\title{
CARMENES Instrument Overview
}

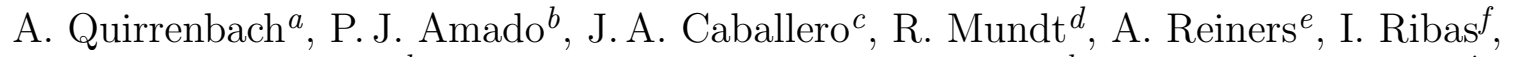
W. Seifert ${ }^{a}$, M. Abril $^{b}$, J. Aceituno ${ }^{g}$, F. J. Alonso-Floriano ${ }^{h}$, M. Ammler-von Eiff ${ }^{i}$, R. Antona Jiménez ${ }^{b}$, H. Anwand-Heerwart ${ }^{e}$, M. Azzaro ${ }^{g}$, F. Bauer ${ }^{e}$, D. Barrado ${ }^{g}$, S. Becerril $^{b}$, V. J.S. Béjar ${ }^{j}$, D. Benítez ${ }^{g}$, Z. M. Berdiñas ${ }^{b}$, M. C. Cárdenas ${ }^{b}$, E. Casal $^{b}$, A. Claret $^{b}$, J. Coloméf, M. Cortés-Contreras ${ }^{h}$, S. Czesla ${ }^{k}$, M. Doellinger ${ }^{i}$, S. Dreizler ${ }^{e}$, C. Feiz $^{a}$, M. Fernández ${ }^{b}$, D. Galadíg ${ }^{\prime}$ M. C. Gálvez-Ortiz ${ }^{c}$, Á. García-Piquer ${ }^{f}$, M. L. García-Vargas ${ }^{l}$, R. Garrido ${ }^{b}$, L. Gesa ${ }^{f}$, V. Gómez Galera ${ }^{g}$, E. González-Álvarez ${ }^{h}$, J. I. González Hernández ${ }^{j}$, U. Grözinger ${ }^{d}$, J. Guàrdia ${ }^{f}$, E. W. Guenther ${ }^{i}$, E. de Guindos ${ }^{g}$, J. Gutiérrez-Soto ${ }^{b}$, H.-J. Hagen ${ }^{k}$, A. P. Hatzes ${ }^{i}$, P. H. Hauschildt ${ }^{k}$, J. Helmling ${ }^{g}$, T. Henning ${ }^{d}$, D. Hermann ${ }^{d}$,

L. Hernández Castaño ${ }^{g}$, E. Herrero ${ }^{f}$, D. Hidalgo ${ }^{h}$, G. Holgado ${ }^{h}$, A. Huber $^{d}$, K. F. Huber ${ }^{k}$,

S. Jeffers ${ }^{e}$, V. Joergens ${ }^{d}$, E. de Juan ${ }^{g}$, M. Kehr ${ }^{i}$, R. Klein ${ }^{d}$, M. Kürster ${ }^{d}$, A. Lamert ${ }^{e}$, S. Lalitha ${ }^{k}, \mathrm{~W} . \mathrm{Laun}^{d}$, U. Lemke ${ }^{e}, \mathrm{R}$. Lenzen $^{d}, \mathrm{M}$. López del Fresno ${ }^{c}$, B. López Martíc J. López-Santiago ${ }^{h}$, U. Mall ${ }^{d}$, H. Mandel ${ }^{a}$, E. L. Martín ${ }^{c}$, S. Martín-Ruiz $^{b}$, H. Martínez-Rodríguez ${ }^{h}$, C. J. Marvin ${ }^{e}$, R. J. Mathar ${ }^{d}$, E. Mirabet ${ }^{b}$, D. Montes ${ }^{h}$, R. Morales Muñoz ${ }^{b}$, A. Moya $^{c}$, V. Naranjo ${ }^{d}$, A. Ofir ${ }^{e}$, R. Oreiro ${ }^{b}$, E. Pallé $^{j}$, J. Panduro $^{d}$, V.-M. Passegger ${ }^{e}$, A. Pérez-Calpena ${ }^{l}$, D. Pérez Medialdea ${ }^{b}$, M. Perger $^{f}$, M. Pluto $^{i}$, A. Ramón ${ }^{b}$,

R. Rebolo ${ }^{j}$, P. Redondo ${ }^{j}$, S. Reffert ${ }^{a}$, S. Reinhardt ${ }^{g}$, P. Rhode $^{e}$, H.-W. Rix ${ }^{d}$, F. Rodler ${ }^{d}$,

E. Rodríguez ${ }^{b}$, C. Rodríguez-López ${ }^{b}$, E. Rodríguez-Pérez ${ }^{b}$, R.-R. Rohloff ${ }^{d}$, A. Rosich ${ }^{f}$ E. Sánchez-Blanco ${ }^{b}$, M. A. Sánchez Carrasco ${ }^{b}$, J. Sanz-Forcada ${ }^{c}$, L. F. Sarmiento ${ }^{e}$, S. Schäfer ${ }^{e}$, J. Schiller ${ }^{i}$, C. Schmidt ${ }^{e}$, J.H. M. M. Schmitt ${ }^{k}$, E. Solano ${ }^{c}$, O. Stahl ${ }^{a}$, C. Storz ${ }^{d}$, J. Stürmer ${ }^{a}$, J. C. Suárez ${ }^{b}$, R.-G. Ulbrich ${ }^{e}$, G. Veredas ${ }^{a}$, K. Wagner ${ }^{a}$, J. Winkler $^{i}$, M. R. Zapatero Osorio $^{c}$, M. Zechmeister ${ }^{e}$, F. J. Abellán de Paco ${ }^{h}$, G. Anglada-Escudém ${ }^{m}$ C. del Burgo ${ }^{n}$, A. Klutsch ${ }^{o}$, J. L. Lizon ${ }^{p}$, M. López-Morales ${ }^{q}$, J. C. Morales ${ }^{r}$, M. A. C. Perryman ${ }^{s}$, S.M. Tulloch' ${ }^{l}$, and W. $\mathrm{Xu}^{t}$

${ }^{a}$ Landessternwarte (LSW), Zentrum für Astronomie der Universität Heidelberg, Königstuhl 12, D-69117 Heidelberg, Germany;

${ }^{b}$ Instituto de Astrofísica de Andalucía (CSIC), Glorieta de la Astronomía s/n, E-18008 Granada, Spain;

${ }^{c}$ Centro de Astrobiología (CSIC-INTA), Campus ESAC, PO Box 78, E-28691 Villanueva de la Cañada, Madrid, Spain;

dMax-Planck-Institut für Astronomie (MPIA), Königstuhl 17, D-69117 Heidelberg, Germany;

${ }^{e}$ Institut für Astrophysik (IAG), Friedrich-Hund-Platz 1, D-37077 Göttingen, Germany; fInstitut de Ciències de l'Espai (CSIC-IEEC), Campus UAB, Facultat Ciències, Torre C5 parell - 2a planta, E-08193 Bellaterra, Barcelona, Spain;

${ }^{g}$ Calar Alto Observatory (MPG-CSIC), Centro Astronómico Hispano-Alemán (CAHA), Jesús Durbán Remón, 2-2, E-04004 Almería, Spain;

${ }^{h}$ Departamento de Astrofísica, Facultad de Física, Universidad Complutense de Madrid, E-28040 Madrid, Spain;

${ }^{i}$ Thüringer Landessternwarte Tautenburg (TLS), Sternwarte 5, D-07778 Tautenburg, Germany; ${ }^{j}$ Instituto de Astrofísica de Canarias, Vía Láctea s/n, E-38205 La Laguna, Tenerife, Spain, and

Dept. Astrofísica, Universidad de La Laguna (ULL), E-38206 La Laguna, Tenerife, Spain;

${ }^{k}$ Hamburger Sternwarte, Gojenbergsweg 112, D-21029 Hamburg, Germany;

${ }^{l}$ FRACTAL SLNE, Tulipán 2, p. 13, 1A, E-28231 Las Rozas, Madrid, Spain; 


\author{
${ }^{m}$ School of Physics and Astronomy, Queen Mary, University of London, 327 Mile End Road, \\ London, E1 4NS, UK; \\ ${ }^{n}$ Instituto Nacional de Astrofísica, Óptica y Electrónica, Luis Enrique Erro 1, Tonantzintla, \\ 72840 Puebla, Mexico; \\ ${ }^{o} \mathrm{INAF}$ - Osservatorio Astrofisico di Catania, via S. Sofia 78, 95123 Catania, Italy; \\ ${ }^{p}$ European Organisation for Astronomical Research in the Southern Hemisphere, \\ Karl-Schwarzschild-Str. 2, D-85748 Garching bei München, Germany; \\ ${ }^{q}$ Harvard-Smithsonian Center for Astrophysics, 60 Garden Street, Cambridge, MA 02138, \\ USA; \\ ${ }^{r}$ LESIA, Observatoire de Paris, Section de Meudon, 5 place Jules Janssen, 92195 Meudon \\ Cedex, France; \\ ${ }^{s}$ School of Physics, University College Dublin, Belfield, Dublin 4, Ireland; \\ ${ }^{t}$ Wenli Xu Optical System Engineering, Kirchenstr. 6, D-74937 Spechbach, Germany
}

\begin{abstract}
This paper gives an overview of the CARMENES instrument and of the survey that will be carried out with it during the first years of operation. CARMENES (Calar Alto high-Resolution search for $\mathbf{M}$ dwarfs with Exoearths with Near-infrared and optical Echelle Spectrographs) is a next-generation radial-velocity instrument under construction for the $3.5 \mathrm{~m}$ telescope at the Calar Alto Observatory by a consortium of eleven Spanish and German institutions. The scientific goal of the project is conducting a 600-night exoplanet survey targeting $\sim 300 \mathrm{M}$ dwarfs with the completed instrument.

The CARMENES instrument consists of two separate échelle spectrographs covering the wavelength range from 0.55 to $1.7 \mu \mathrm{m}$ at a spectral resolution of $R=82,000$, fed by fibers from the Cassegrain focus of the telescope. The spectrographs are housed in vacuum tanks providing the temperature-stabilized environments necessary to enable a $1 \mathrm{~m} / \mathrm{s}$ radial velocity precision employing a simultaneous calibration with an emission-line lamp or with a Fabry-Pérot etalon. For mid-M to late-M spectral types, the wavelength range around $1.0 \mu \mathrm{m}(Y$ band) is the most important wavelength region for radial velocity work. Therefore, the efficiency of CARMENES has been optimized in this range.

The CARMENES instrument consists of two spectrographs, one equipped with a $4 k \times 4 k$ pixel CCD for the range $0.55-1.05 \mu \mathrm{m}$, and one with two $2 k \times 2 k$ pixel $\mathrm{HgCdTe}$ detectors for the range from $0.95-1.7 \mu \mathrm{m}$. Each spectrograph will be coupled to the $3.5 \mathrm{~m}$ telescope with two optical fibers, one for the target, and one for calibration light. The front end contains a dichroic beam splitter and an atmospheric dispersion corrector, to feed the light into the fibers leading to the spectrographs. Guiding is performed with a separate camera; on-axis as well as off-axis guiding modes are implemented. Fibers with octagonal cross-section are employed to ensure good stability of the output in the presence of residual guiding errors. The fibers are continually actuated to reduce modal noise. The spectrographs are mounted on benches inside vacuum tanks located in the coudé laboratory of the $3.5 \mathrm{~m}$ dome. Each vacuum tank is equipped with a temperature stabilization system capable of keeping the temperature constant to within $\pm 0.01^{\circ} \mathrm{C}$ over 24 hours. The visible-light spectrograph will be operated near room temperature, while the near-IR spectrograph will be cooled to $\sim 140 \mathrm{~K}$.
\end{abstract}

The CARMENES instrument passed its final design review in February 2013. The MAIV phase is currently ongoing. First tests at the telescope are scheduled for early 2015. Completion of the full instrument is planned for the fall of 2015. At least 600 useable nights have been allocated at the Calar Alto $3.5 \mathrm{~m}$ Telescope for the CARMENES survey in the time frame until 2018.

A data base of M stars (dubbed CARMENCITA) has been compiled from which the CARMENES sample can be selected. CARMENCITA contains information on all relevant properties of the potential targets. Dedicated

Further author information: Send correspondence to A. Quirrenbach

E-mail: A.Quirrenbach@lsw.uni-heidelberg.de, Telephone: +49-6221-541792 
imaging, photometric, and spectroscopic observations are underway to provide crucial data on these stars that are not available in the literature.

Keywords: Spectrographs, Optical Instrumentation, Near-Infrared Instrumentation, Extrasolar Planets, M Dwarfs

\section{THE GOALS OF CARMENES}

Radial-velocity (RV) surveys of $\mathrm{M}$ dwarfs are gaining momentum as an important complement to surveys of more massive stars, and as a method to discover and possibly characterize hot and temperate rocky exoplanets. M-type dwarfs, with masses in the range of $0.1-0.6 M_{\odot}$, are the most abundant type of stars in our Galaxy (frequency $\sim 70 \%$ ), and therefore obtaining statistics of planetary system occurrence and architecture for these stars is of great importance to understand the physics of planet formation and evolution in general, and its dependence on stellar host mass. Radial-velocity searches for planets around M dwarfs benefit from a larger signal, and a shorter orbital period of planets in the habitable zone (HZ). These advantages, along with the larger transit depths, have been exploited to find some of the low-mass exoplanets known so far, both with the radial-velocity method (e.g., Mayor et al. 2009, Anglada-Escudé et al. 2012), and with transits (Charbonneau et al. 2009).

However, the current number of planet detections with M-star hosts is still low compared with solar analogs. Therefore the abundance of planets as a function of mass and orbital distance is not well constrained at the moment, and the much-sought value of $\eta_{\oplus}$, i.e., the abundance of Earth-analog planets in the HZ, still has a large uncertainty. In addition, present RV surveys cover only M-type stars of spectral types earlier than M2 or M3, corresponding to $M_{*} \geq 0.3$ to $0.4 M_{\odot}$. The faintness of the targets in the visible wavelength range and the intrinsic stellar jitter have up to now limited the investigation of stars with even lower masses.

The CARMENES (Calar Alto high-Resolution search for $\mathbf{M}$ dwarfs with Exo-earths with Near-infrared and optical Echelle Spectrographs; see also Quirrenbach et al. 2010, 2012) project is aimed at filling this gap, by constructing a radial-velocity instrument optimized for planet searches of mid- to late-type $\mathrm{M}$ dwarfs. The main scientific driver for CARMENES is the search for very low-mass planets (i.e., Earth-analogs and "super-Earths") around mid- to late-type $\mathrm{M}$ dwarfs, and around moderately active $\mathrm{M}$ dwarfs in general. Specifically, we aim at a long-term RV precision and stability of $1 \mathrm{~m} \mathrm{~s}^{-1}$ per measurement, sufficient to detect a $2 M_{\oplus}$ planet in the middle of the $\mathrm{HZ}$ of an M5 V star. For stars later than $\sim \mathrm{M} 4\left(M<0.25 M_{\odot}\right)$, such precision will yield detections of super-Earths of $5 M_{\oplus}$ and smaller inside the entire width of the HZ.

Our survey strategy is to intensively monitor a well-characterized sample of about $300 \mathrm{M}$ dwarfs. We can estimate the percentage of stars with low-mass planets by extrapolating current results of planet surveys to lower stellar masses. If we assume that about $30-40 \%$ of the $\mathrm{M}$ dwarfs have low-mass planets and a high chance of some being in the habitable zone, we may expect to find between 50 and 100 suitable planets. This estimate is obviously rather uncertain, but even if the real frequency is much lower, still a substantial number of detections will be available, and CARMENES will put tight constraints on the abundance and orbital parameters of low-mass planets in the investigated mass regime.

The expected CARMENES planet harvest is, at any rate, sufficient to carry out a reliable statistical analysis of the planet population and shed light on the architecture of planetary systems and on their formation mechanisms. The CARMENES survey will also provide valuable constraints on $\eta_{\oplus}$ for $\mathrm{M}$ dwarfs. Given the transit probability and the favorable selection bias, there is a good chance of finding a few transiting low-mass planets in the habitable zone. These hold extraordinary value for future investigations since they will be orbiting some of the nearest stars in the solar neighborhood and thus their discovery merits substantial efforts.

After completion of the primary CARMENES survey anticipated for the end of 2018, the instrument will be positioned well to provide powerful follow-up capabilities for space-borne transit missions such as TESS (Ricker et al. 2014) and PLATO (Rauer et al. 2014). 


\section{THE CARMENES INSTRUMENT}

A set of simulations has been carried out to determine the best wavelength range for RV observations of M dwarfs (Reiners et al. 2010). The results strongly argue in favor of a solution with wide wavelength coverage from the visible to the near-infrared. This will yield the highest possible precision while permitting at the same time the discrimination of planet detections against false-positive RV signals caused by stellar activity. Activity-induced RV variations are expected to be wavelength dependent, which is strictly not the case for orbital variations. Data show that the wavelength dependence of activity-induced RV signals will result in at least a factor of 2 to 3 different amplitude in the range $0.55-1.7 \mu \mathrm{m}$, and thus provide an efficient and safe way to discard spurious signals. This range is therefore adopted for the CARMENES instrument.

A fair number of $\mathrm{M}$ dwarfs have rotational velocities $(v \sin i)$ of less than 3 to $4 \mathrm{~km} / \mathrm{s}$. For these stars, rather high spectral resolution is beneficial for precise RV measurements. For CARMENES, a requirement of $R \approx 82,000$ has been adopted, as the modest gain in RV precision of even higher resolution does not justify the additional cost.

To meet the scientific goals of the CARMENES survey, the instrument has been designed to provide a stability of $1 \mathrm{~m} / \mathrm{s}$. This requirement has been applied to the specifications of all sub-systems.

\subsection{System Overview}

For mid-M to late-M spectral types, the wavelength range around $1.0 \mu \mathrm{m}$ ( $Y$ band) is the most important wavelength region for RV work. Therefore, the efficiency of CARMENES has been optimized in this range. Since CCDs do not provide high enough efficiency around $1.0 \mu \mathrm{m}$ and no signal at all beyond the silicon cutoff at $1.1 \mu \mathrm{m}$, a near-IR detector is required. It is thus natural to adopt an instrument concept with two spectrographs, one equipped with a CCD for the range $0.55-1.05 \mu \mathrm{m}$, and one with $\mathrm{HgCdTe}$ detectors for the range $0.95-$ $1.7 \mu \mathrm{m}$.

Each spectrograph will be coupled to the $3.5 \mathrm{~m}$ telescope with its own optical fiber. The front end contains a dichroic beam splitter and an atmospheric dispersion corrector, to feed the light into the fibers leading to the spectrographs. Guiding is performed with a separate camera. Additional fibers are available for simultaneous injection of light from emission line lamps for RV calibration, or, alternatively, from the sky.

The two spectrographs have been designed to be as similar to each other as possible. They are mounted on benches inside vacuum tanks, which are located in climatic chambers inside the coudé laboratory of the $3.5 \mathrm{~m}$ dome. The near-IR vacuum tank is equipped with a temperature stabilization system; the thermal design of both spectrographs provides the capability of keeping the temperature of the optical bench constant to within $\pm 0.01 \mathrm{~K}$ over $24 \mathrm{~h}$. The visible-light spectrograph will be operated near room temperature, the near-IR spectrograph will be cooled to $\sim 140 \mathrm{~K}$.

\subsection{Front End}

The CARMENES front end is attached to the Cassegrain focus of the telescopes and provides the following functionalities: mechanical interface to the telescope, target acquisition and guiding, compensation of the atmospheric dispersion, separation of visible and near-infrared light, switching between calibration fibers and the sky, shutter for the visible-light channel, and injection of the light into optical fibers that connect to the spectrographs.

The optical design of the front end consists of a flat $90^{\circ}$ pick-up mirror followed by an atmospheric dispersion compensator (ADC). The pick-up mirror can be retracted to let the light pass through to a different instrument (e.g., the PMAS integral-field spectrograph, with which the front end is fully compatible). The light coming from the telescope is then divided by a dichroic mirror to feed the science fibers for the visible-light and, via an additional fold mirror, the near-IR spectrographs. The guiding camera uses the light reflected back from a mirror in front of the visible-light fiber head.

An ADC is needed in front of the dichroic plate in order to correct for differential displacement of the light rays across the wide wavelength band caused by the Earth's atmosphere. The ADC has therefore to cover the complete wavelength range of $0.55-1.7 \mu \mathrm{m}$. This has been realized by a two double-prisms design to provide nearly full correction to the atmospheric dispersion. The double-prism is made of Ohara glasses S-BAL12 and 


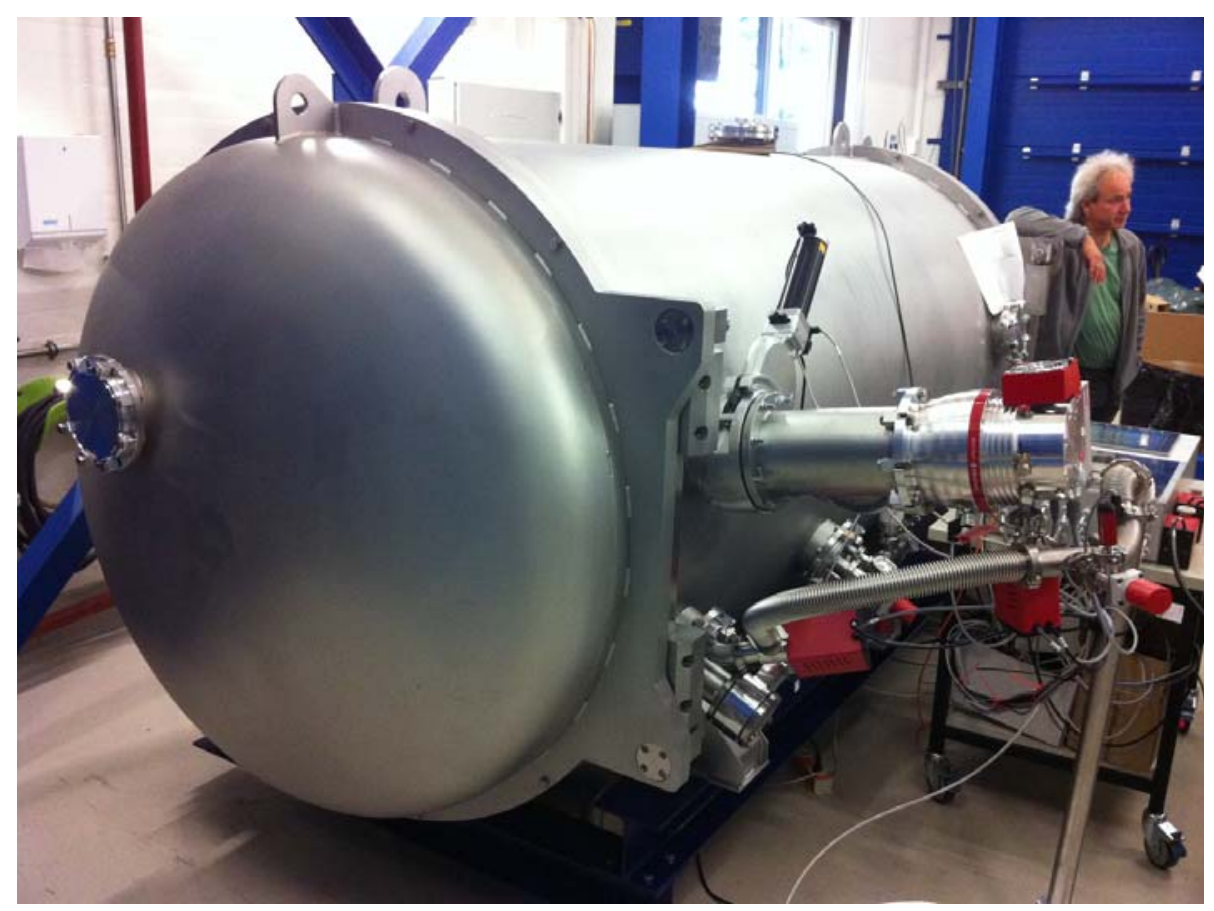

Figure 1. The vacuum tank for the visible-wavelength spectrograph, integrated with vacuum pumps and control system in Heidelberg. The tank has an inner diameter of $1400 \mathrm{~mm}$ and a length of $3500 \mathrm{~mm}$; it was manufactured by NTG (Gelnhausen, Germany). The vacuum control system development within the CARMENES consortium has been led by CAHA. The nearly identical vacuum system for the near-IR spectrograph is being integrated in Granada.

SFTM16, which have very good internal transmission over the whole wavelength range. The residual dispersion is less than $5 \mu \mathrm{m}$ (i.e., small compared to the fiber diameter) across the whole wavelength band up to a zenith distance of $70^{\circ}$. The two double-prisms have to be counter-rotated for each observation to align it with respect to the horizon.

\subsection{Optical Fibers}

CARMENES uses 14 optical fibers, i.e., seven for each of the two channels (see Stürmer et al. 2014 for details). All fibers are of the type CeramOptec Optran WF and consist of a pure silica core and a fluorine-doped silica cladding. For the whole wavelength range of CARMENES their attenuation is less than $10 \mathrm{~dB} / \mathrm{km}$. The longest fibers in CARMENES are around $50 \mathrm{~m}$. Therefore the internal losses are always smaller than $5 \%$ for both channels.

Each of the two spectrographs is connected to the front end with a science and a reference fiber, which are identical for the two spectrograph arms. Each of these fibers consists of a circular section and an octagonal section. The long, circular part runs from the front end down to the temperature-stabilized room. There it is connected to a shorter octagonal part, which is fed to the spectrograph. The fiber connection is an FC-FC junction, allowing for a relatively easy replacement of the science or the reference fiber if one of them gets damaged during operation; the spectrograph does not have to be opened for replacement.

The fibers do not only transport the light from the telescope to the spectrograph, but they also provide for a stable input illumination of the spectrographs. Changing the illumination in the near field (which is the illumination right at the fiber surface) is known to have the biggest impact on radial velocity precision. The use of octagonal fibers minimizes this effect. However, the stability of the far field (which is the angular distribution of the light exiting the fiber) is also important, because a change in the angular light distribution leads to a change of illumination of the grating and other optics. Due to imperfections and aberrations this leads to changes of the point spread function of the whole instrument. Extensive lab tests and simulations of these effects have 


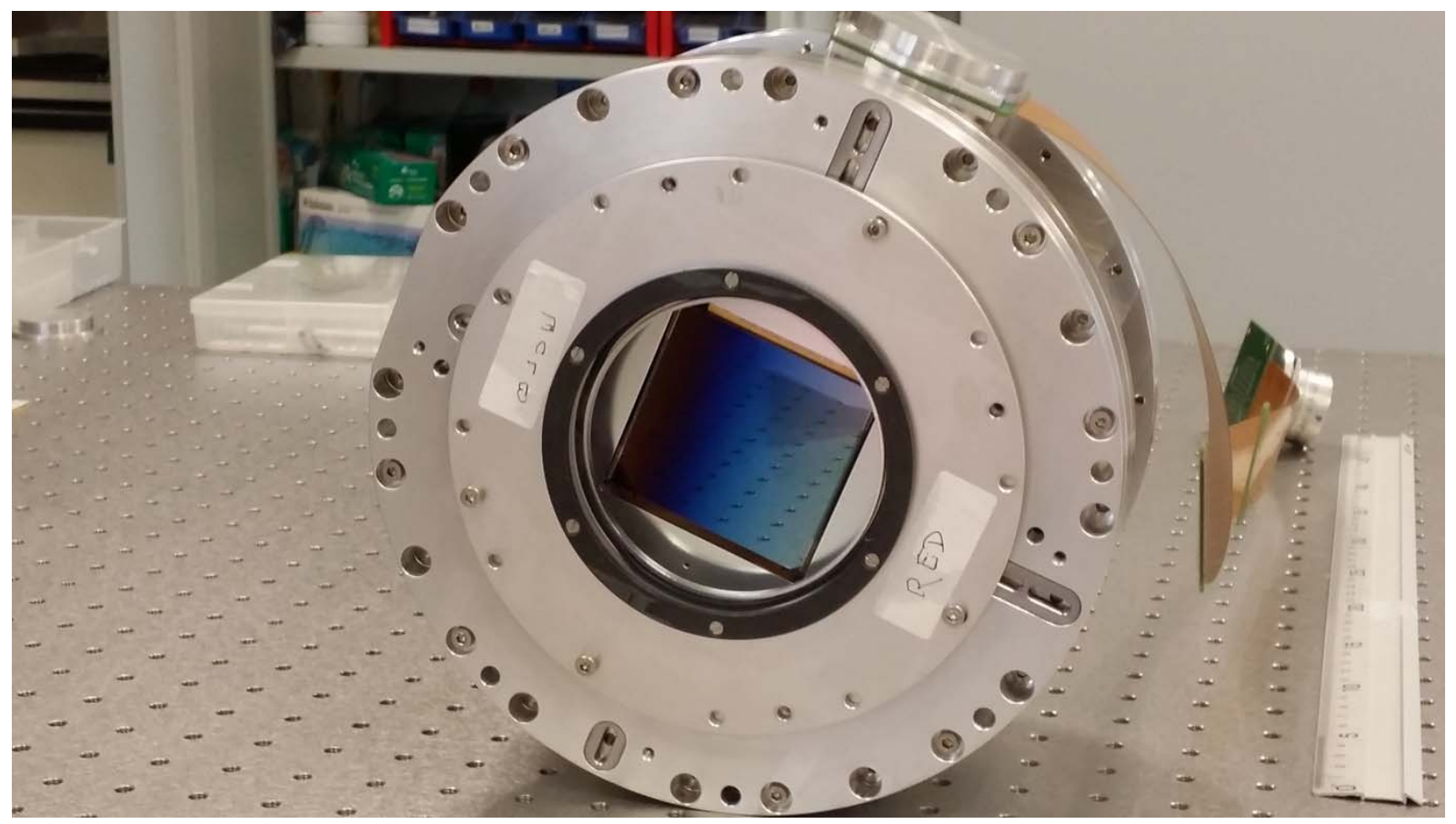

Figure 2. The visible-light detector (e2v CCD231-84) integrated in its cryostat at the LICA observatory in Madrid.

been conducted to ensure that they remain below the $1 \mathrm{~m} / \mathrm{s}$ level. To reduce modal noise, the fibers are also agitated mechanically during the exposure (Stürmer et al. 2014).

Additional fibers are used to transport light from the calibration units to the front end, and from a pick-up inside the spectrograph to a real-time exposure meter for each channel.

\subsection{Visible-Light Spectrograph}

The CARMENES spectrographs are based on an échelle design with grism cross-dispersion and a white pupil, working in quasi-Littrow mode (Seifert et al. 2012). To keep the beam size manageable, each spectrograph contains an image slicer that accepts two beams (from the science and reference fibers), and divides their pupils into two slices. The visible-light spectrograph covers the wavelength range $0.55-1.05 \mu \mathrm{m}$ with a resolving power of $R=82,000$ and a mean sampling of 2.8 pixels per resolution element (ppre). Due to strong anamorphism introduced by the R4 échelle, the sampling varies strongly along each order, from 2.3 ppre at the blue end to 4.0 ppre at the red end of the free spectral range in the most extreme case. The spectrograph is mounted on a bench inside a vacuum vessel (see Fig. 1). The detector is a back-side illuminated $4112 \times 4096$ pixel CCD supplied by $\mathrm{e} 2 \mathrm{v}$ (model CCD231-84). The visible-light spectrograph is operated near room temperature, with an anticipated temperature stability of $\pm 0.01{ }^{\circ} \mathrm{C}$ within 24 hours. There are no moving parts inside the vacuum vessel to maximize mechanical stability and to avoid heat dissipation.

Since CARMENES requires very good thermal and mechanical stability, the use of mechanical coolers, which would cause vibrations and instability, is precluded. Liquid nitrogen thus remains the best choice for cooling the detector. As for a number of ESO instruments, a continuous flow of liquid nitrogen has been chosen to provide the cooling. The continuous flow system offers a number of advantages compared to a bath cryostat: longer hold time without intervention, consequently less human activity around the instrument, no change of mass during operation, and storage of the nitrogen supply in a commercial tank external to the instrument. The continuous flow cryostats for CARMENES have been developed with the help of ESO; the CCD is shown within its cryostat in Fig. 2. 


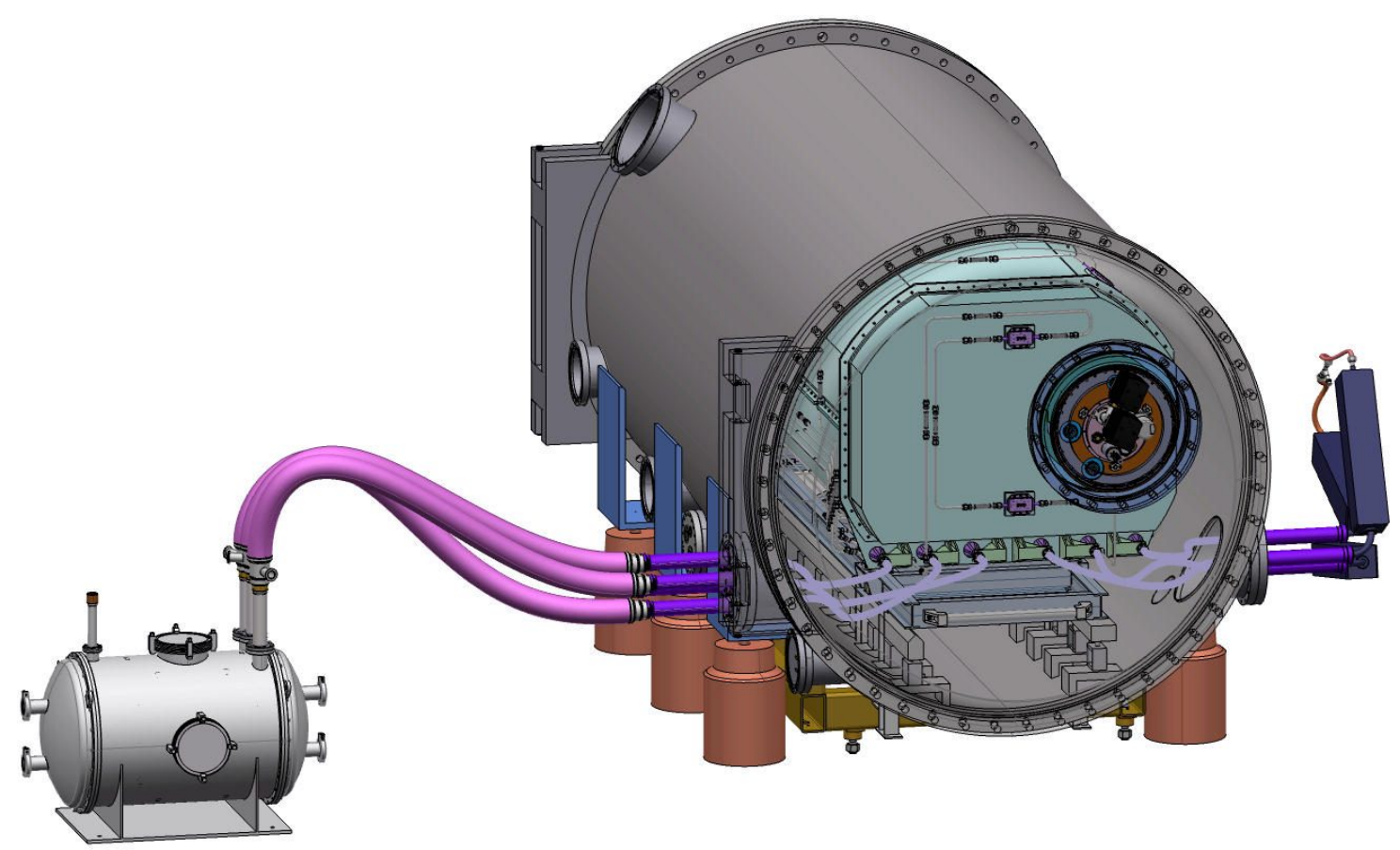

Figure 3. Sketch of the nitrogen gas preparation unit (N2GPU) connected to the near-infrared vacuum tank.

\subsection{Near-Infrared Spectrograph}

The optical and mechanical design of the near-IR spectrograph is very similar to that of its visible counterpart. The near-IR spectrograph provides $R=82,000$ over the wavelength range $0.95-1.7 \mu \mathrm{m}$ with a mean sampling of 2.5 ppre. Since CARMENES does not use any light longward of $1.7 \mu \mathrm{m}$, it would be convenient to employ near-IR detectors with a cut-off near that wavelength. However, whereas some detectors with that cut-off have been delivered and tested, they have not yet reached the technical and operational maturity of devices with a "standard" $2.5 \mu \mathrm{m}$ cut-off. For that reason, the detector of the near-IR channel in CARMENES is a mosaic of two $2048 \times 2048$ pixel HAWAII-2RG infrared arrays with a long-wavelength cutoff at $2.5 \mu \mathrm{m}$ (see also Amado et al. 2012). With this choice of detector, it is necessary to cool the spectrograph to $140 \mathrm{~K}$.

The near-IR cooling system employs an external heat exchanger / evaporator unit that is fed by liquid nitrogen and provides a continuous flow of gaseous nitrogen to the near-IR spectrograph (Becerril et al. 2012, Mirabet et al. 2014; see also Fig. 3). This nitrogen gas is coupled with heat exchangers to a radiation shield within the spectrograph tank. The stabilization of the shield, together with the large thermal inertia of the optical bench, ensures excellent stability (to within $\pm 0.01 \mathrm{~K}$ over 24 hours) of the optical system.

\subsection{Calibration Units}

CARMENES uses hollow-cathode emission line lamps (Th-Ne for the visible channel, U-Ne in the near-IR) for spectral calibration (Sarmiento et al. 2014). This simultaneous reference method requires a series of calibration exposures before the beginning of the night. During the night, the "daily" calibration lamps remain switched on in parallel to the science observations. They are not used to produce a wavelength solution but to trace the differential nightly drift of the spectrograph. This leads to a significant duty cycle of these lamps. The limited lifetime of hollow-cathode lamps requires that the lamps for nightly operation are calibrated regularly against "master" lamps that are used less frequently. The wavelength solution used for science observations will be determined from these "master" lamps. The procedure to produce a wavelength solution for both fibers is carried out every day. This calibration schedule will eventually also lead to degradation of the master lamps. The calibration unit will therefore contain three master lamps: one to be used every day, a second to be used once per week in addition to the first lamp, and a third lamp to take over if the first lamp fails. An additional 

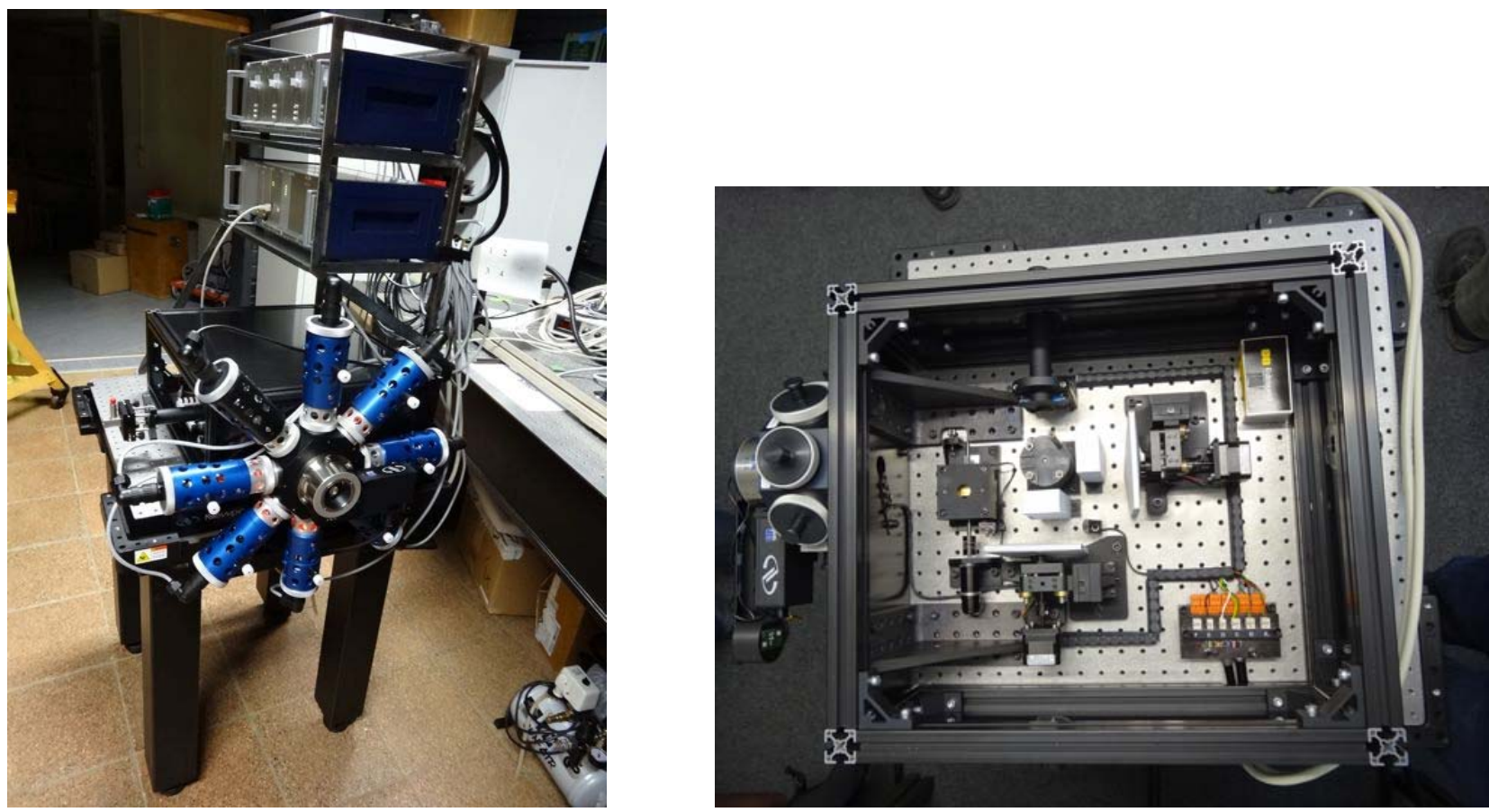

Figure 4. Calibration unit for the visible channel built by TLS. The housings for eight lamps in an octagonal arrangement are visible in the foreground of the picture on the left. In the right picture, the cover has been removed to show the optics feeding the calibration light into the fibers. External ports that can accept fibers from other calibration sources (e.g., etalons) are also available. The near-IR calibration unit is nearly identical.

wavelength solution from this reserve lamp should be produced twice a year. The master lamps should also be calibrated twice per year against another set of "super-master" lamps.

For each spectrograph, the arc lamps as well as quartz lamps for flat-fielding are housed in a calibration unit (see Fig. 4), which is connected to the front end with a fiber link. The lamps can be switched on and off independently; a rotating mirror is used to switch between the lamps. Care has been taken to make this mechanism light-tight, so that several lamps can be on (and thus at a stable operating temperature) at any given time without disturbing the exposure being taken. This enables fast switching between the lamps for the crosscalibrations described above. The super-master lamps are stored in a dedicated tank filled with a low-pressure neon atmosphere. The calibration units also provide additional input ports for Fabry-Pérot etalons, which may provide a better method for tracking the spectrograph drifts during the night.

\subsection{Control System and Data Reduction Pipeline}

The coordination and management of the sub-systems of CARMENES is handled by the instrument control system (ICS), which provides a tool to operate the instrument in an integrated manner (Guàrdia et al. 2012, García-Piquer et al. 2014). The ICS interacts with the following subsystems: the detectors and exposure meters of the near-IR and visible channels, the calibration units, the environmental sensors, the front-end electronics, the acquisition and guiding module, the interfaces with telescope and dome, and, finally, the software subsystems for operational scheduling of tasks, data processing, and data archiving. The CARMENES operational scheduler is the second key element in the control layer; it is the main actor in the translation of the survey strategy into a detailed schedule. The scheduler is based on artificial intelligence techniques and plans the survey by combining the static constraints that are known a priori (e.g., target visibility, sky background, moon phase and position, required time sampling coverage) and the dynamic changes (e.g., weather, system conditions). This is expected to increase the efficiency of telescope the operations, which will represent an important benefit in terms of scientific return and operational costs. 


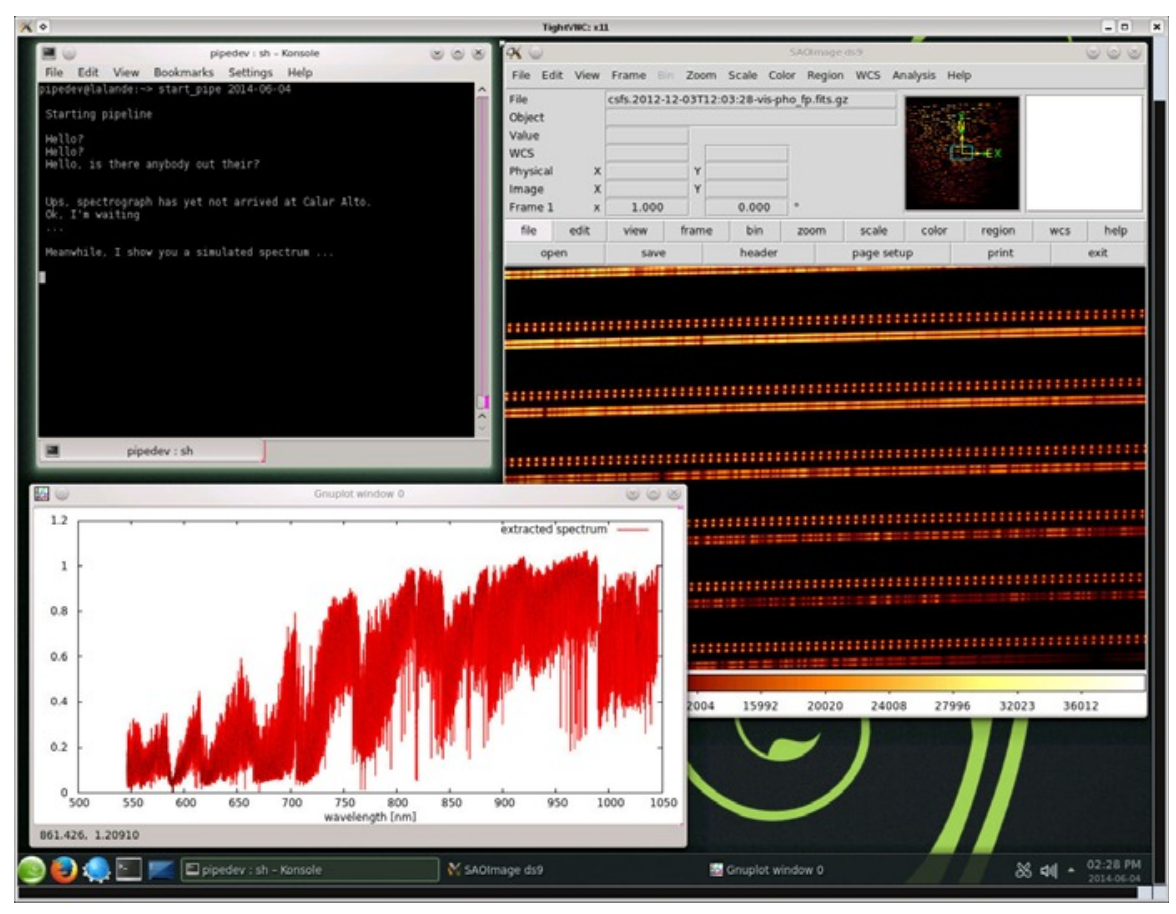

Figure 5. Screenshot of one of the pipeline computers, showing a spectrum extracted from simulated échelle data.

After completion of an exposure, the data will be displayed and forwarded to an on-line version of the data reduction pipeline. This pipeline will allow a first run of the data reduction during the night to constantly monitor integrity and data quality. This will allow immediate response to changing weather conditions and to quick identification of problems. During the next day, science quality data reduction and radial velocity measurements will be performed after all calibration exposures are taken. A screenshot of the user interface of one of the pipeline computers is shown in Fig. 5.

\section{PREPARING FOR THE CARMENES SURVEY}

To prepare for a proper selection and characterization of our targets, we have created the CARMENCITA database (CARMENes Cool star Information and daTa Archive), in which we are consolidating information available in the literature (Quirrenbach et al. 2012, Caballero et al. 2013). We are also collecting additional data (e.g., low-resolution spectra, where not available), and performing homogeneous analyses to obtain spectral types and other stellar parameters (Klutsch et al. 2012, Alonso-Floriano et al. 2013). In this context, we have obtained low-resolution $(R \sim 1500)$ spectra of $752 \mathrm{M}$ (and late $\mathrm{K}$ ) dwarfs fainter than $J=9$ mag with CAFOS at the $2.2 \mathrm{~m}$ Calar Alto telescope. For all of them, we have derived spectral types with 0.5 subtypes accuracy. We have also obtained metallicity and surface gravity from spectral indices, and activity from the $\mathrm{H} \alpha$ equivalent width. Next, we have observed $522 \mathrm{M}$ dwarfs at high-resolution $(R=30,000$ to 48,000) with FEROS at the ESO/MPG $2.2 \mathrm{~m}$ on La Silla, CAFE at the $2.2 \mathrm{~m}$ on Calar Alto, and with HRS at Hobby Eberly Telescope. We have determined for the first time the projected rotational velocity $v \sin i$ (with accuracy \pm 0.2 to $0.5 \mathrm{~km} / \mathrm{s}$ ) and radial velocity (to \pm 0.1 to $0.2 \mathrm{~km} / \mathrm{s}$ ) of 414 stars. Our observations allow us to identify high-activity, lowmetallicity and low-gravity stars, single- and double-lined spectroscopic binaries and, especially, fast rotators, which should be discarded from any target list for exoplanet searches.

We are also investigating the multiplicity of the stars in CARMENCITA. Our multiplicity study covers a wide range in projected physical separations, from 0.5 to 55,000 AU. The inner range is covered with a lucky-imaging survey of $385 \mathrm{M}$ dwarfs with FastCam at the $1.5 \mathrm{~m}$ Telescopio Carlos Sánchez (42.3 mas/pix), complemented with a literature search. We have explored visual or physical companions from 0". 15 to $18^{\prime \prime}$ around our targets. These observations are important to discard very close companions that may induce spurious variations in the 


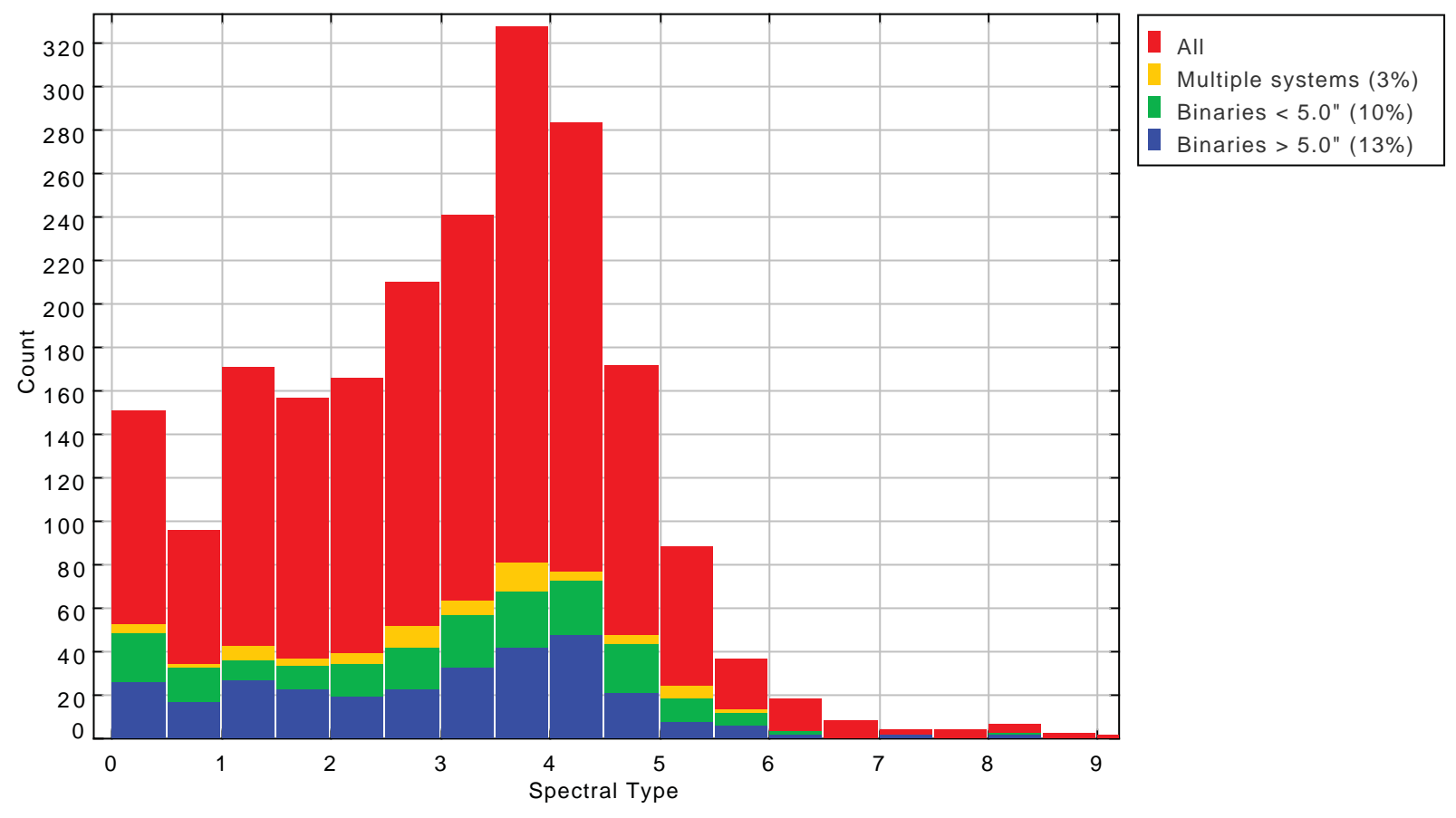

Figure 6. Spectral type distribution of close and wide binaries and multiple systems (more than two components) in CARMENCITA (2131 M dwarfs as of March 2014). The x-axis is the spectral sub-type within the M spectral class. The total multiplicity fraction is $26 \%$, in agreement with similar numbers given by Janson et al. (2012, 2014) and Jódar et al. (2013).

radial velocity of the primary and mimic the presence of planets. The outer range is covered with a detailed analysis of Washington Double Stars catalog data and optical images taken by us with TCP and CAMELOT at the $0.8 \mathrm{~m}$ IAC80 telescope, and an astrometric study of all-sky public images and catalogs. The result of these analyses are summarized in Fig. 6.

At the start of the CARMENES survey, we will observe 450 stars; after further "cleaning" (i.e., removal of unsuitable targets after a few initial observations), we aim at arriving at three sub-samples of 100 stars each:

- S1: $M<0.25 M_{\odot}$ (spectral type M4 V and later);

- S2: $0.25 M_{\odot}<M<0.30 M_{\odot}($ spectral type M3 V - M4 V);

- S3: $0.30 M_{\odot}<M<0.60 M_{\odot}$ (spectral type M0 V - M2 V), relatively bright.

Sample S1 is designed to cover the spectral type domain that is very faint in the visible and can only be studied by CARMENES. Sample S2 is selected to address a pool of targets for which CARMENES is very efficient but comparable to visible spectrographs, so that a cross-check with other surveys is possible. Sample S3 will have the highest fraction of bright targets and will therefore be best suited as a "poor weather" sample. Taken together, studying all three samples will provide a comprehensive view of the planet population around low-mass stars.

For the final core sample of 300 stars we plan to obtain at least 60 measurements per object, and at least 100 measurements or more for the most interesting systems. The experience gathered with similar surveys indicates that many exoplanets can already be identified reliably with about 30 measurements. However, $\sim 100$ measurements per object are likely needed for the detection of a planet with RV semi-amplitude close to the measurement error, and to discern multiple-planet systems, which seem to be quite abundant when composed of mainly low-mass planets. We will thus have to acquire $~ 22,500$ spectra to achieve the goals of the CARMENES survey. 
To observe the sample we will use a typical maximum integration time of $900 \mathrm{~s}$, guaranteeing a signal-to-noise ratio of 150 for targets with $J=9$ mag. When considering the expected instrument overheads this leads to an estimate of some 3.5 measurements per hour or some 30 measurements per night, thus adding up to about 750 clear nights.

\section{OUTLOOK: BRINGING CARMENES TO THE TELESCOPE}

After passing the final design review (FDR) in February 2013, CARMENES is now in the manufacturing, assembly, integration, and verification (MAIV) phase. Several examples have been shown above for components and subsystems that are currently being assembled and tested. The front end and the visible spectrograph are being integrated at LSW and MPIA in Heidelberg; the near-IR spectrograph is undergoing integration at IAA in Granada. After full testing of the completed systems at these locations, they will be shipped to Calar Alto and re-integrated at the observatory. The preparation of the coudé room of the $3.5 \mathrm{~m}$ telescope for CARMENES is proceeding in parallel. We expect that we can perform initial on-sky tests of the front end in early 2015, in order to verify the acquisition procedures and guiding performance. The two spectrographs will be commissioned in the course of 2015, so that the CARMENES survey with the full instrument can star at the beginning of 2016 .

\section{ACKNOWLEDGMENTS}

CARMENES is an instrument for the Centro Astronómico Hispano-Alemán de Calar Alto (CAHA, Almería, Spain). CARMENES is funded by the German Max-Planck-Gesellschaft (MPG), the Spanish Consejo Superior de Investigaciones Científicas (CSIC), the European Union through FEDER/ERF funds, and the members of the CARMENES Consortium (see institutions ${ }^{a}$ through ${ }^{k}$ of the author list), with additional contributions by the Spanish Ministry of Economy, the state of Baden-Württemberg, the German Science Foundation (DFG), and by the Junta de Andalucía.

\section{REFERENCES}

1. Alonso-Floriano, F.J., Montes, D., Caballero, J.A., Klutsch, A., Morales, J.C., et al. (2013). CARMENES. IV. Preliminary low-resolution spectroscopic characterisation. In Highlights of Spanish astrophysics VII, proceedings of the $X$ scientific meeting of the Spanish Astronomical Society (SEA). Eds. Guirado, J.C., Lara, L.M., Quilis, V., \& Gorgas, J., p. 431

2. Amado, P.J., Lenzen, R., Cardenas, M.C., Sánchez-Blanco, E., Becerril, S., et al. (2012). CARMENES: non-cryogenic solutions for YJH-band NIR instruments. In Modern technologies in space-and ground-based telescopes and instrumentation II. Eds. Navarro, R., Cunningham, C.R., \& Prieto, E., SPIE 84501U

3. Anglada-Escudé, G., Arriagada, P., Vogt, S.S., Rivera, E.J., Butler, R.P., et al. (2012). A planetary system around the nearby M Dwarf GJ 667 c with at least one super-Earth in its habitable zone. ApJ 751, L16

4. Becerril, S., Lizon, J.L., Sánchez-Carrasco, M.A., Mirabet, E., Amado, P., et al. (2012). CARMENES. III: an innovative and challenging cooling system for an ultrastable NIR spectrograph. In Modern technologies in space- and ground-based telescopes and instrumentation II. Eds. Navarro, R., Cunningham, C.R., \& Prieto, E., SPIE 84504L

5. Caballero, J.A., Cortés-Contreras, M., López-Santiago, J., Alonso-Floriano, F.J., Klutsch, A., et al. (2013). CARMENES. III. CARMENCITA, the input catalogue. In Highlights of Spanish astrophysics VII, proceedings of the X scientific meeting of the Spanish Astronomical Society (SEA). Eds. Guirado, J.C., Lara, L.M., Quilis, V., \& Gorgas, J., p. 645

6. Charbonneau, D., Berta, Z.K., Irwin, J., Burke, C.J., Nutzman, P., et al. (2009). A super-Earth transiting a nearby low-mass star. Nature 462, 891-894

7. García-Piquer, A., Guàrdia, J., Colomé, J., Ribas, I., Gesa, L., \& Morales, J.C. (2014). CARMENES Instrument Control System and Operational Scheduler. These proceedings, 9152-72

8. Guàrdia, J., Colomé, J., Ribas, I., Hagen, H.J., Morales, R., et al. (2012). CARMENES. IV: instrument control software. In Software and cyberinfrastructure for astronomy II. Eds. Radziwill, N.M., \& Chiozzi, G., SPIE $84512 \mathrm{~S}$ 
9. Janson, M., Hormuth, F., Bergfors, C., Brandner, W., Hippler, S., et al. (2012). The AstraLux large M-dwarf multiplicity survey. ApJ 754, 44

10. Janson, M., Bergfors, C., Brandner, W., Kudryavtseva, N., Hormuth, F., et al. (2014). The AstraLux multiplicity survey: extension to late M-dwarfs. ApJ 789, 102

11. Jódar, E., Pérez-Garrido, A., Díaz-Sánchez, A., Villó, I., Rebolo, R., \& Pérez-Prieto, J.A., (2013). New companions to nearby low-mass stars. MNRAS 429, 859-867

12. Klutsch, A., Alonso-Floriano, F.J., Caballero, J.A., Montes, D., Cortés-Contreras, M., et al. (2012). Spectral characterisation of the CARMENES input catalogue. In SF2A-2012: Proceedings of the annual meeting of the French Society of Astronomy and Astrophysics. Eds. Boissier, S., de Laverny, P., Nardetto, N., Samadi, R., Valls-Gabaud, D., \& Wozniak, H., p. 357-360

13. Mayor, M., Bonfils, X., Forveille, T., Delfosse, X., Udry, S., et al. (2009). The HARPS search for southern extra-solar planets. XVIII. An Earth-mass planet in the GJ 581 planetary system. A\&A 507, 487-494

14. Mirabet, E., Carvas, P., Lizon, J.L., Becerril, S., Amado., P.J., et al. (2014). CARMENES in SPIE 2014: Very promising results about an ultrahigh stability cooling system for the CARMENES instrument. These Proceedings, 9151-150

15. Quirrenbach, A., Amado, P.J., Mandel, H., Caballero, J.A., Mundt, R., et al. (2010). CARMENES: Calar Alto high-Resolution search for $M$ dwarfs with Exo-earths with Near-infrared and optical Echelle Spectrographs. In Ground-based and airborne instrumentation for astronomy III. Eds. McLean, I.S., Ramsay, S.K., \& Takami, H., SPIE 773513

16. Quirrenbach, A., Amado, P.J., Seifert, W., Sánchez Carrasco, M.A., Mandel, H., et al. (2012). CARMENES. I: instrument and survey overview. In Ground-based and airborne instrumentation for astronomy IV. Eds. McLean, I.S., Ramsay, S.K., \& Takami, H., SPIE 84460R

17. Rauer, H., Catalá, C., Aerts, C., Appourchaux, T., Benz, W., et al. (2014). The PLATO 2.0 Mission. ExA, in press

18. Reiners, A., Bean, J.L., Huber, K.F., Dreizler, S., Seifahrt, A., \& Czesla, S. (2010). Detecting planets around very low mass stars with the radial velocity method. ApJ 710, 432-443

19. Ricker, G.R., Winn, J.N., Vanderspek, R., Latham, D.W., Bakos, G.A., et al. (2014). The Transiting Exoplanet Survey Satellite. These Proceedings, 9143-508

20. Sarmiento, L.F., Reiners, A., Seemann, U., Lemke, U., Winkler, J., et al. (2014). Characterizing U-Ne hollow cathode lamps at near-IR wavelengths for the CARMENES survey. These proceedings, 9147-194

21. Seifert, W., Sánchez-Carrasco, M., Xu, W., Cárdenas, C., Sánchez-Blanco, E., et al. (2012). CARMENES. II: optical and opto-mechanical design. In Ground-based and airborne instrumentation for astronomy IV. Eds. McLean, I.S., Ramsay, S.K., \& Takami, H., SPIE 844633

22. Stürmer, J., Stahl, O., Schwab, C., Seifert, W., Quirrenbach, A., et al. (2014). CARMENES in SPIE 2014. Building a fiber link for CARMENES. These proceedings, 9151-191 